\section{Revista de CIENCIAS AMBIENTALES Tropical Journal of Environmental Sciences}

Revista de Ciencias Ambientales (Trop J Environ Sci) e-ISSN: 2215-3896

(Enero-Junio, 2022) . Vol 56(1): 120-137

DOI: https://doi.org/10.15359/rca.56-1.6

Open Access: www.revistas.una.ac.cr/ambientales e-mail: revista.ambientales@una.ac.cr Rodríguez Cueto Y., Ramón Puebla A.

\title{
Evaluación temporal y espacial en la calidad microbiológica del agua superficial: caso en un sistema de abastecimiento de agua para consumo humano en Costa Rica
}

\author{
Temporal and spatial assessment of the surface water microbiology quality: A case \\ in a human consumption water supply system in Costa Rica
}

\author{
Eric Morales-Mora ${ }^{1}$, Liliana Reyes-Lizano ${ }^{2}, K_{\text {Kenia Barrantes-Jiménez }}^{3}$, \\ Luz Chacón-Jiménez ${ }^{4}$
}

[Recibido: 28 de enero 2021, Aceptado: 21 de septiembre 2021, Corregido: 06 de octubre 2021, Publicado: 1 de enero 2022]

\begin{abstract}
Resumen
[Introducción]: El agua para consumo es un determinante de la salud humana; particularmente cuando se obtiene de fuentes superficiales puede ser afectada por contaminantes microbiológicos causantes de enfermedades, los cuales a su vez son influenciados por las condiciones ambientales; sin embargo, estos efectos han sido poco estudiados en Costa Rica. [Objetivo]: Determinar la influencia de variables ambientales en las concentraciones de microorganismos patógenos e indicadores fecales en un sistema de abastecimiento de agua para consumo humano. [Metodología]: Se realizó un muestreo dos veces al mes durante un año. Se midió la concentración de Cryptosporidium sp., Giardia sp. colifagos somáticos y Escherichia coli. Se analizaron las condiciones espaciales mediante el uso de sistemas de información geográfica, y las condiciones climáticas de manera descriptivas. Para el análisis de datos, se utilizaron técnicas para datos censurados y un modelo de regresión de Poisson para evaluar la influencia de la intensidad de precipitación sobre las concentraciones de microorganismos. [Resultados]: Se encuentran diferencias estacionales para colifagos somáticos y E. coli. Cryptosporidium sp. y Giardia sp. son más frecuentes cuando la intensidad de precipitación es débil, mientras que colifagos somáticos y $E$. coli presentan mayores concentraciones conforme aumenta la intensidad de precipitación. [Conclusiones]: Las cuatro microcuencas presentan vulnerabilidad por contaminación con respecto a sus condiciones espaciales y se determina que tanto la estacionalidad climática y la intensidad de la precipitación $24 \mathrm{~h}$ antes afectan de manera diferente a cada uno de los microorganismos en estudio, por lo cual, se recomienda su evaluación periódica de forma independientemente.
\end{abstract}

Palabras clave: calidad del agua; estaciones del año; intensidad de precipitación; salud ambiental.

1 Especialista en Salud Ambiental, Instituto de Investigaciones en Salud, Universidad de Costa Rica, Costa Rica; eric.morales@ucr.ac.cr; https://orcid.org/0000-0001-8090-4947

2 Microbióloga, Instituto de Investigaciones en Salud, Universidad de Costa Rica, Costa Rica; lilireyesli@gmail.com; https://orcid. org/0000-0002-6722-184X

3 Microbióloga, Instituto de Investigaciones en Salud, Universidad de Costa Rica, Costa Rica; kenia.barrantes@ucr.ac.cr; https://orcid. org/0000-0002-2673-9220

4 Microbióloga, Instituto de Investigaciones en Salud, Universidad de Costa Rica, Costa Rica; luz.chacon@ucr.ac.cr; https://orcid. org/0000-0003-2506-0619

(C)

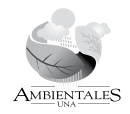




\section{Revista de CIENCIAS AMBIENTALES Tropical Journal of Environmental Sciences}

Revista de Ciencias Ambientales (Trop J Environ Sci) e-ISSN: 2215-3896

(Enero-Junio, 2022) . Vol 56(1): 120-137 DOI: https://doi.org/10.15359/rca.56-1.6 Open Access: www.revistas.una.ac.cr/ambientales e-mail: revista.ambientales@una.ac.cr Rodríguez Cueto Y., Ramón Puebla A.

\begin{abstract}
[Introduction]: Drinking water is a determining factor in human health; when obtained from surface sources, it can be affected by microbiological contaminants that cause disease, which in turn could be influenced by environmental conditions. However, these effects have been little studied in Costa Rica. [Objective]: To determinate the environmental variability influence in the concentrations of pathogen microorganisms and fecal indicators in a drinking water system. [Methodology]: samples were collected twice a month for one year. Cryptosporidium sp., Giardia sp., somatic coliphages, and Escherichia coli concentrations were measure. Spatial conditions were analyzed using geographic information systems, and climate conditions analysis were performed using descriptive technics. To data analysis were used censored data technics and Poisson regression models to evaluate the influence of the rainfall on microorganisms' concentrations. [Results]: seasonal differences were found for somatic coliphages and E. coli. Cryptosporidium sp. and Giardia sp. concentrations were more frequent with weak precipitation intensity. Meanwhile, coliphages and E. coli concentrations were higher when precipitation intensity increase. [Conclusions]: The four micro-basins presented pollution vulnerability for their spatial conditions. Besides, study microorganisms were affected differently by climatic seasonality and $24 \mathrm{~h}$ previous intensity of the precipitation. Therefore, it is recommended an individual evaluation of them periodically.
\end{abstract}

Keywords: environmental health; precipitation intensity; seasons; water quality.

\title{
1. Introducción
}

La calidad del agua se considera uno de los pilares principales para el desarrollo sostenible, por su rol en la salud humana y su utilidad en la ejecución de actividades fundamentales para la sociedad. Uno de los más importantes riesgos de contaminación para este recurso son los microorganismos patógenos que pueden ingresar a los cuerpos de agua a través de descargas directas e indirectas de materia fecal y aguas residuales (World Health Organitation, WHO, 2017). De acuerdo con la WHO (2017), la ingesta de aguas contaminadas, la falta de higiene y condiciones de sanidad inseguras fueron responsables de alrededor de un millón de muertes a nivel global, de las cuales 470000 se dieron por diarrea en menores de cinco años.

La transmisión hídrica es una de las vías más comúnmente utilizadas por los patógenos para diseminarse. Por ejemplo, Cryptosporidium sp. y Giardia sp., dos de los protozoarios patógenos más frecuentes a nivel mundial, utilizan esta vía de diseminación, ya que poseen características que permiten este tipo de transmisión, por ejemplo, bajas dosis infectantes, permanecen viables tras el tratamiento convencional del agua para consumo humano y tienen alta resistencia a la cloración, aunado a que entre sus principales reservorios se encuentran los bovinos y animales domésticos que pueden estar en contacto fácilmente con cuerpos de agua superficial (Chalmers, 2014; Robertson, 2014). Escherichia coli, por su parte, además de ser uno de los indicadores más tradicionales de contaminación microbiológica del agua, es el microorganismo del grupo coliforme más común en el ser humano y es susceptible a la cloración; a pesar de ello, su transmisión hídrica es relevante debido a que algunas de sus cepas son patógenas para el ser humano, siendo las diarreogénicas de particular importancia en esta vía (Strachan et al., 2006; WHO, 2017). Por último, los virus entéricos son otro grupo de patógenos transmitidos por agua, uno

\begin{tabular}{|c|c|c|c|c|}
\hline 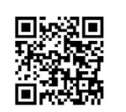 & (c) (1) (9) & $\underset{\text { AMBEFINALES }}{\infty}$ & $\frac{O \%}{\text { euna }}$ & 121 \\
\hline
\end{tabular}




\section{Revista de CIENCIAS AMBIENTALES Tropical Journal of Environmental Sciences}

Revista de Ciencias Ambientales (Trop J Environ Sci) e-ISSN: 2215-3896 (Enero-Junio, 2022) . Vol 56(1): 120-137 DOI: https://doi.org/10.15359/rca.56-1.6 Open Access: www.revistas.una.ac.cr/ambientales e-mail: revista.ambientales@una.ac.cr Rodríguez Cueto Y., Ramón Puebla A.

de los indicadores de contaminación fecal viral más reconocidos son los colifagos somáticos. Estos bacteriófagos son virus que infectan E. coli intestinal y su detección en aguas se asocia a la presencia de virus entéricos capaces de provocar enfermedad diarreica en seres humanos (Chacón et al., 2020; McMinn et al., 2017).

La movilidad de contaminantes microbiológicos, como los ya mencionados, hacia ambientes acuáticos es favorecida por condiciones ambientales como la altitud y pendiente, el uso de suelo y fluctuaciones temporales en la precipitación, entre otras (Bergion et al., 2017). Mientras que el uso del suelo urbano, ganadero o agrícola, cerca de los cuerpos de agua, puede facilitar la contaminación con materia fecal; una densa cobertura vegetal puede prevenirla. Las fluctuaciones climáticas e hidrológicas pueden modificar la distribución y frecuencia de los patógenos en el ambiente promoviendo, en algunos casos, el contacto entre un hospedero y un agente infeccioso (Bertuzzo \& Mari, 2017). Por último, condiciones geomorfológicas, como las altas pendientes, favorecen la escorrentía, lo cual aumenta la movilización de los patógenos hacia los ríos, costas o pozos (Lal et al., 2013).

Teniendo en cuenta estos antecedentes en el presente estudio, se analizó la relación de los parámetros espaciales y temporales de cuatro microcuencas utilizadas en la producción de agua para consumo humano, con respecto a la calidad microbiológica del agua de acuerdo con las concentraciones de: Cryptosporidium sp., Giardia sp., E. coli y colifagos somáticos. Con este fin, se consideraron parámetros espaciales y temporales como: densidad de vegetación, uso de suelo, geomorfología y clima (comportamiento anual y diario). En concordancia con lo anterior, los objetivos de este estudio son: describir espacial y temporalmente las zonas de captación en estudio mediante el uso de sistemas de información geográfica y un climograma; evaluar el comportamiento espacial y estacional de microorganismos en estudio mediante análisis de varianza y temporales; analizar la posible asociación estadística de la precipitación diaria y las concentraciones de microorganismos en estudio; generar recomendaciones para la gestión y el manejo del recurso hídrico utilizado en la producción de agua para uso humano, considerando variables espaciales y temporales.

\section{Metodología}

\subsection{Zona de estudio y sitios de muestreo}

El estudio se realizó entre febrero de 2017 y marzo de 2018 en cuatro microcuencas. Los puntos de muestreo se ubicaron en cada río, antes de la entrada a la zona de cribado correspondiente a cada una de las cuatro captaciones de un sistema de abastecimiento de agua para consumo humano, ubicado al este del Gran Área Metropolitana de Costa Rica (Figura 1). Este sistema fue seleccionado debido a que abastece aproximadamente 70000 personas $(1.4 \%$ de la población costarricense). Se encuentra ubicado en la parte alta de la cuenca del Río Grande de Tárcoles, en las provincias de San José y Cartago. Es importante indicar que, en este sistema

\begin{tabular}{|c|c|c|}
\hline 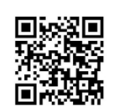 & (c) () () () & 122 \\
\hline
\end{tabular}




\section{Revista de CIENCIAS AMBIENTALES Tropical Journal of Environmental Sciences}

Revista de Ciencias Ambientales (Trop J Environ Sci) e-ISSN: 2215-3896 (Enero-Junio, 2022) . Vol 56(1): 120-137 DOI: https://doi.org/10.15359/rca.56-1.6 Open Access: www.revistas.una.ac.cr/ambientales e-mail: revista.ambientales@una.ac.cr Rodríguez Cueto Y., Ramón Puebla A.

particular, las captaciones se encuentran varios kilómetros corrientes abajo de la naciente y que los puntos de muestreo se establecieron como punto de menor altitud en cada microcuenca. La zona de estudio se clasifica bioclimáticamente como bosque muy húmedo montano bajo, con excepción de la parte alta de la microcuenca A, clasificada como bosque pluvial montano. El suelo es tipo andisol, el cual se caracteriza por su alta permeabilidad. La clasificación bioclimática y de suelos fue realizada con la superposición de capas vectoriales provenientes del Atlas de Costa Rica (Ortiz-Malavasi, 2014).

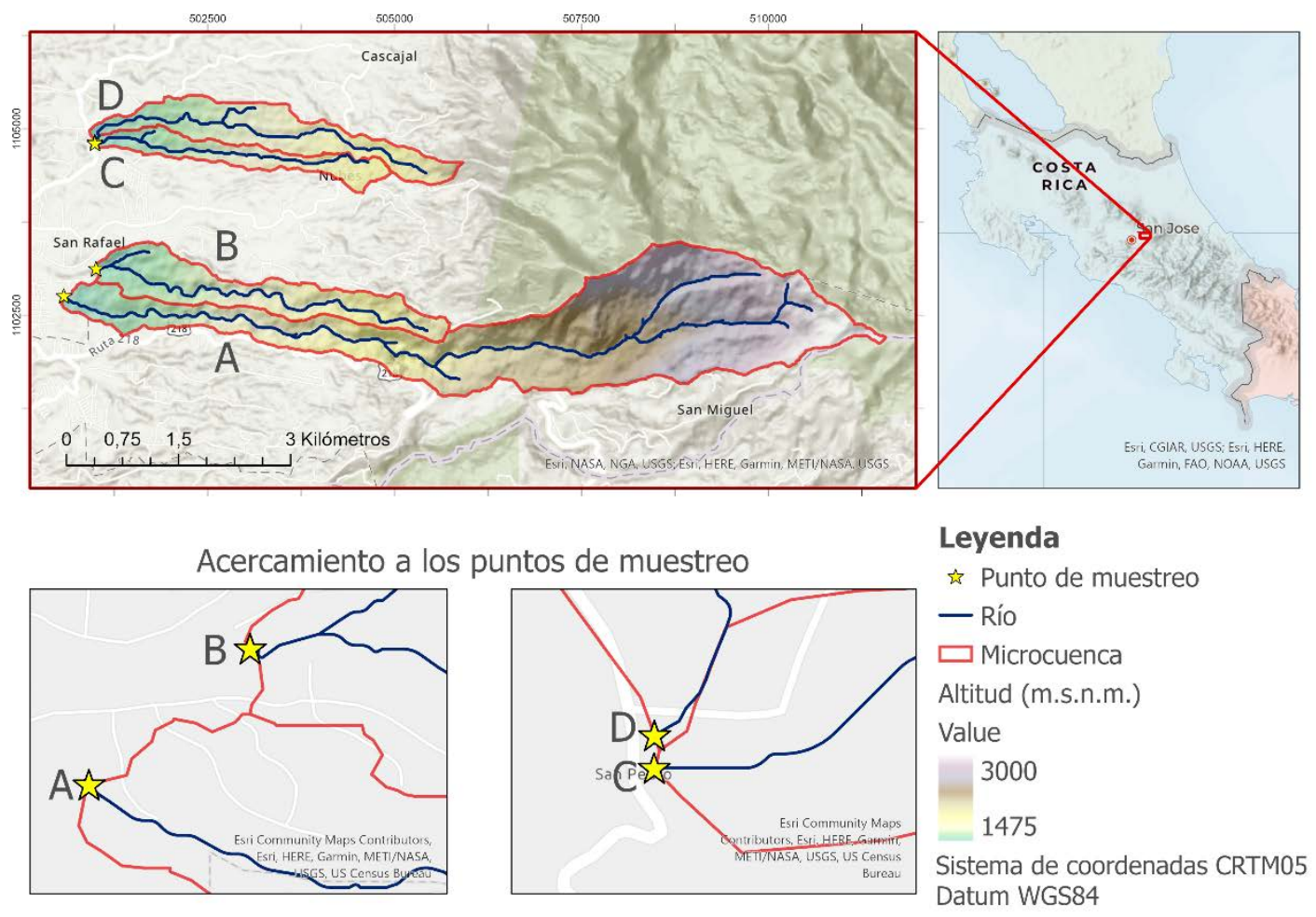

Figura 1. Ubicación de la zona de estudio.

Figure 1. Location of the study area.

Cada captación (A, B, C y D para efectos de este estudio) corresponde a una microcuenca, cuya delimitación se describe en Morales et al. (2019) (Figura 1). Se realizó un muestreo a lo largo de 54 semanas (incluyendo época seca y lluviosa), dos veces al mes (cada 15 días), para un total de 108 muestras analizadas. La toma y transporte de las muestras se realizó de acuerdo al Standard Methods for the Examination of Water and Wastewater (SMEWW) (Baird, 2017).

\begin{tabular}{|c|c|c|c|c|}
\hline 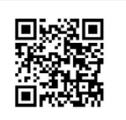 & (c) (i) (9) & 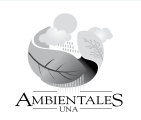 & $\frac{1 \% \%}{\text { euna }}$ & 123 \\
\hline
\end{tabular}




\section{Revista de CIENCIAS AMBIENTALES Tropical Journal of Environmental Sciences}

Revista de Ciencias Ambientales (Trop J Environ Sci) e-ISSN: 2215-3896 (Enero-Junio, 2022) . Vol 56(1): 120-137 DOI: https://doi.org/10.15359/rca.56-1.6 Open Access: www.revistas.una.ac.cr/ambientales e-mail: revista.ambientales@una.ac.cr Rodríguez Cueto Y., Ramón Puebla A.

\subsection{Descripción espacial}

La descripción ambiental se divide en dos componentes: espacial y temporal. Para la descripción espacial en las microcuencas se analizó: densidad de vegetación, pendiente, morfología y uso de suelo; para todos los análisis, se utilizó el software ArcGIS 10.8 (ESRI software). Los datos para la estimación de la densidad de vegetación y el uso de suelo fueron obtenidos del proyecto Lansat-8 en la plataforma del Servicio Geológico de los Estados Unidos Earth Explorer (http:// earthexplorer.usgs.gov/). La densidad y calidad de la vegetación fue determinada utilizando el índice de vegetación de diferencia normalizada (NDVI, por sus siglas en inglés), el cual es una medida del crecimiento de la vegetación derivada de la proporción de la reflectancia entre las bandas del rojo (R) e infrarrojo cercano (NIR) de imágenes compuestas que detectan absorbancia de la clorofila (Pettorelli et al., 2005), su cálculo se realizó como se muestra a continuación:

$$
N D V I=\frac{N I R-R}{N I R+R}
$$

El NDVI oscila entre -1 y 1 , tradicionalmente se clasifica de la siguiente manera: - 1 ausencia de vegetación o vegetación muerta, $0.0-\leq 0.3$ vegetación enferma, $>0.3-\leq 0.6$ vegetación medianamente sana y $>0.6-1.0$ vegetación muy sana (Pettorelli et al., 2005). Sin embargo, también hay cabida a otras clasificaciones, el NDVI más allá de la descripción de la salud y densidad de la vegetación permite la diferenciación de otros objetos espaciales, por ejemplo, las rocas desnudas, arena, nieve, edificaciones o suelo desnudo tienden a presentar valores menores a 0.1 . La vegetación escasa, arbustos o pastizales tienden a presentar valores entre $0.2-0.5$, mientras altos valores (0.6-0.9) corresponden a vegetación muy densa como bosques templados y tropicales o cosechas en su pico de crecimiento (Pettorelli et al., 2005; USGS, 2021). Este indicador es una herramienta con gran potencial para aplicaciones ambientales, puesto que además de ser un descriptor de la salud y densidad de la vegetación, también puede ser utilizado como descripción de uso de suelo, análisis de la variabilidad ecológica, evaluación de ganancias de carbono, diferenciación entre tipos de bosque (primario o secundario), descriptor del mantenimiento de la humedad en el suelo y predictor de la diseminación de microorganismos patógenos (Cabello et al., 2016; Jagai et al., 2009; Valle et al., 2019).

Para la delimitación del uso de suelo, se utilizaron imágenes satelitales para generar composiciones espectrales, las cuales facilitan el reconocimiento de patrones espaciales. En este estudio se utilizaron las composiciones de color natural e infrarrojo cercano-infrarrojo de onda corta-rojo. La clasificación del uso de suelo se realizó utilizando aprendizaje automático supervisado con el algoritmo de clasificación por el método de estimación de máxima verosimilitud (Dheenan et al., 2016). Adicionalmente, se realizaron visitas de campo para verificar y definir usos de suelo desconocidos.

Para la estimación de la morfología y pendiente, se utilizó un modelo de elevación digital (DEM) con una resolución de 12.5 m x 12.5 m del proyecto Alaska Satellite Facility de la NASA

\begin{tabular}{|c|c|c|c|c|}
\hline 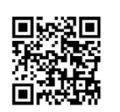 & (c) (1) (9) & $\underset{\text { AMBIENALIES }}{\longrightarrow}$ & $\frac{10 \%}{\text { euna }}$ & 124 \\
\hline
\end{tabular}




\section{Revista de CIENCIAS AMBIENTALES Tropical Journal of Environmental Sciences}

Revista de Ciencias Ambientales (Trop J Environ Sci) e-ISSN: 2215-3896 (Enero-Junio, 2022) . Vol 56(1): 120-137 DOI: https://doi.org/10.15359/rca.56-1.6 Open Access: www.revistas.una.ac.cr/ambientales e-mail: revista.ambientales@una.ac.cr Rodríguez Cueto Y., Ramón Puebla A.

(Lee, 2020). La morfología fue analizada mediante el uso del DEM y un mapa de sombras para su visualización de manera descriptiva. Para la determinación de la pendiente, se estimaron los grados de inclinación en el terreno.

\subsection{Descripción temporal}

Para definir la estacionalidad, se consideró la clasificación según la época seca y lluviosa definida en Costa Rica, por lo que se analizó la diferencia en la concentración de microorganismos obtenida para cada una de estas estaciones a fin de identificar patrones estacionales de estos. Por otro lado, para el análisis de la influencia de la precipitación sobre las concentraciones de microorganismos, se obtuvieron datos climáticos del Instituto Meteorológico Nacional y se categorizó la intensidad de precipitación correspondiente a la media por hora de las veinticuatro horas previas al muestreo. Las categorías establecidas fueron las siguientes: débil $(\geq 2 \mathrm{~mm} / \mathrm{h})$, moderado $(>2 \mathrm{~mm} / \mathrm{h}-\leq 15 \mathrm{~mm} / \mathrm{h})$, fuerte $(>15 \mathrm{~mm} / \mathrm{h}-\leq 30 \mathrm{~mm} / \mathrm{h})$ y muy fuerte $(>30 \mathrm{~mm} / \mathrm{h}$ - $\leq 60 \mathrm{~mm} / \mathrm{h}$ ); no hubo datos superiores a $60 \mathrm{~mm} / \mathrm{h}$. Para la descripción anual climática, se consideraron las mediciones de precipitación acumulada $(\mathrm{mm} / \mathrm{h})$ y temperatura $\left({ }^{\circ} \mathrm{C}\right)$ media en veinticuatro horas. Para el análisis descriptivo se agregaron los datos por mes y se superpusieron las variables de temperatura y precipitación mensual con el fin de generar un climograma.

\subsection{Análisis microbiológicos}

La detección de Cryptosporidium sp. y Giardia sp. se realizó a partir de la concentración de estos parásitos con el método de floculación con $\mathrm{Al}_{2}\left(\mathrm{SO}_{4}\right)_{3}$ descrito por Karanis et al., (2006). El concentrado obtenido en la floculación fue depurado utilizando la técnica de inmunoseparación magnética con el kit Dynal GC-Combo', según las instrucciones del fabricante (Dynal Biotech ASA, Oslo, Noruega). Las muestras purificadas se colocaron en un pocillo de un portaobjetos y fueron teñidas con isotiocianato de fluoresceína conjugado con anticuerpos monoclonales anti-crypto y anti-giardia, de acuerdo con las instrucciones del fabricante (Feal de Durviz.l., España). Las muestras teñidas fueron observadas en un microscopio de fluorescencia Olympus ${ }^{\circledast}$ CLX modelo BX43F, con una magnitud de onda entre $490-540 \mathrm{~nm}$, se observó todo el pocillo y se realizó el conteo de las formas parasitarias siguiendo lo dispuesto en el método 1623 (US-EPA, 2005). Adicionalmente, cada portaobjeto contaba con un pocillo de control positivo y negativo. El límite de detección para ambos parásitos es $<0,1$ (oo)quistes/L.

La cuantificación de colifagos somáticos se realizó utilizando el método de capa simple de agar descrito por Solano et al., (2012), el cual consiste en mezclar $100 \mathrm{~mL}$ de ATS 1.2 \%, $5 \mathrm{~mL}$ de cloruro de magnesio y $10 \mathrm{~mL}$ de cultivo de E. coli ATCC 13906 en fase logarítmica, con una densidad óptica de $0.300 \pm 0.050$ y $100 \mathrm{~mL}$ de la muestra de agua previamente filtrada por filtros de $47 \mathrm{~mm}$ de acetato de celulosa de $0.2 \mu \mathrm{m}$ de poro (Sartorius, Alemania), previamente tratados con extracto de carne pH 7.2 (Oxoid, Estados Unidos). La mezcla se homogenizó y se dispensó en platos Petri, los cuales se incubaron a $35.0 \pm 0.5^{\circ} \mathrm{C}$ durante veinticuatro horas. Las unidades

\begin{tabular}{|c|c|c|}
\hline 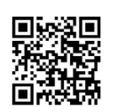 & (c) (i) () & 125 \\
\hline
\end{tabular}




\section{Revista de CIENCIAS AMBIENTALES Tropical Journal of Environmental Sciences}

Revista de Ciencias Ambientales (Trop J Environ Sci) e-ISSN: 2215-3896 (Enero-Junio, 2022) . Vol 56(1): 120-137 DOI: https://doi.org/10.15359/rca.56-1.6 Open Access: www.revistas.una.ac.cr/ambientales e-mail: revista.ambientales@una.ac.cr Rodríguez Cueto Y., Ramón Puebla A.

formadoras de placas se determinaron tras el conteo de los halos translúcidos en las placas Petri. Los límites de detección son: <1 UFP/ 100 mL - >3000 UFP/ 100 mL.

Para la cuantificación de E. coli se utilizó el método del número más probable con la técnica de fermentación en tubos múltiples descrita en el apartado 9221 de SMEWW (Baird, 2017). Para esto, se utilizó como medio de enriquecimiento el caldo lauril triptosa (Oxoid, Estados Unidos) y, como medio selectivo para E. coli, el caldo EC-MUG (Oxoid, Estados Unidos). Para definir una muestra como positiva, se estableció que el tubo debía presentar turbidez, gas y la fluorescencia característica al exponerse a luz ultravioleta (Baird, 2017). La interpretación de los resultados se realizó utilizando la tabla de número más probable y se utilizó como control positivo la E. coli ATCC 25922 y, como control negativo, Pseudomonas aeruginosa 14502. Los límites de detección del método son: $<1.8 \mathrm{NMP} / 100 \mathrm{~mL}$ - >1 600000 NMP/100 mL.

\subsection{Análisis de datos}

El análisis de datos se realizó incluyendo el método no paramétrico de supervivencia y métodos ordinarios para la exploración de datos; además, dada la presencia de datos censurados a la izquierda se utilizó el paquete NADA (Lee, 2020) del lenguaje de programación R de CRAN (Helsel, 2011). Para el estudio, se establecieron dos grupos de análisis: microcuencas (A, B, C, D) y época climática (lluviosa y seca). La selección de método para la estimación de los estadísticos descriptivos se determinó según las características del conjunto de datos: tamaño de la muestra y proporción de datos censurados (Helsel, 2011). Para Cryptosporidium sp., Giardia sp. y colifagos somáticos, se utilizó el método de Kaplan-Meir y Turnbull en los dos grupos de análisis, para la posterior construcción de un diagrama de bigotes (boxplot) con valores censurados entre la época lluviosa (de mayo a noviembre) y la época seca (de diciembre a abril). Para cada una de las variables microbiológicas en estudio, se analizaron las diferencias entre grupos utilizando el método no paramétrico de Peto-Prentice. En el caso de E. coli, para determinar las diferencias entre las zonas de captación, se utilizó el método de Kruskal-Wallis y entre época climática la prueba Wilcoxon.

Para evaluar la relación entre la intensidad de precipitación veinticuatro horas antes del muestreo (categorizada previamente) y la concentración de Cryptosporidium sp., Giardia sp. colifagos somáticos y E. coli, se aplicó una regresión de Poisson bivariada. El análisis fue realizado utilizando el software R con la función glm (generalised linear models) para el análisis de variables continuas en modelos múltiples. Posterior al análisis, se calcula el coeficiente exponencial de la regresión de Poisson para el cálculo de las razones de momios (odd ratio).

Para determinar la bondad del ajuste, se realizó una prueba chi-cuadrado, donde se utilizó la función pchisq(), y para probar la sobre dispersión, se aplicó una prueba de dispersión, utilizando la función dispersiontest() de la librería AER. En ambos casos, para que el modelo sea válido, se debe obtener un valor de p >0.05. Para todos los análisis estadísticos, se utilizó una significancia $\mathrm{p}<0.10$.

\begin{tabular}{|c|c|c|c|c|c|}
\hline 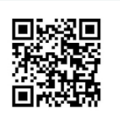 & (c) (i) () & $\overbrace{\text { AMEIENTILES }}$ & $\begin{array}{l}\frac{9 \%}{2} \\
\frac{2}{2} \\
\text { euna }\end{array}$ & 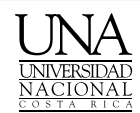 & 126 \\
\hline
\end{tabular}




\section{Revista de CIENCIAS AMBIENTALES Tropical Journal of Environmental Sciences}

Revista de Ciencias Ambientales (Trop J Environ Sci) e-ISSN: 2215-3896 (Enero-Junio, 2022) . Vol 56(1): 120-137 DOI: https://doi.org/10.15359/rca.56-1.6 Open Access: www.revistas.una.ac.cr/ambientales e-mail: revista.ambientales@una.ac.cr Rodríguez Cueto Y., Ramón Puebla A.

\section{Resultados}

\subsection{Descripción espacial}

La zona de estudio tiene un área total de $14.3 \mathrm{~km}^{2}$; la densidad de vegetación, medida a través del NDVI, osciló entre 0.03 (vegetación enferma o muerta) y 0.58 , con una media de 0.33 ; lo cual, de acuerdo con Pettorelli et al. (2005), la clasifica como de vegetación medianamente sana. Cabe destacar que la mayor parte de las zonas con vegetación enferma o nula, se encuentran cerca de los cuerpos de agua, situación más frecuente en la microcuenca A (Figura 2).

\section{1. Índice de vegetación de diferencia normalizada (NDVI)}

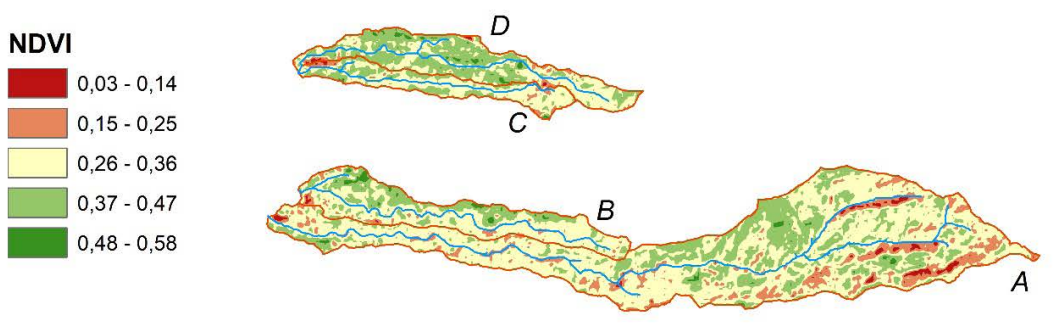

\section{Uso de suelo}

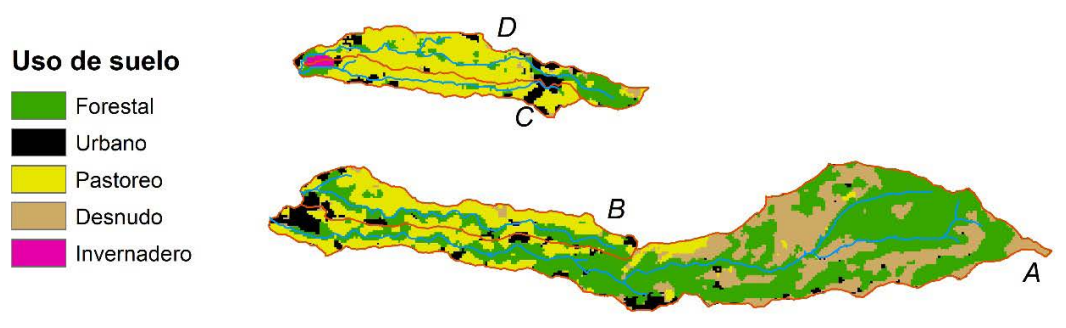

\section{Pendiente}

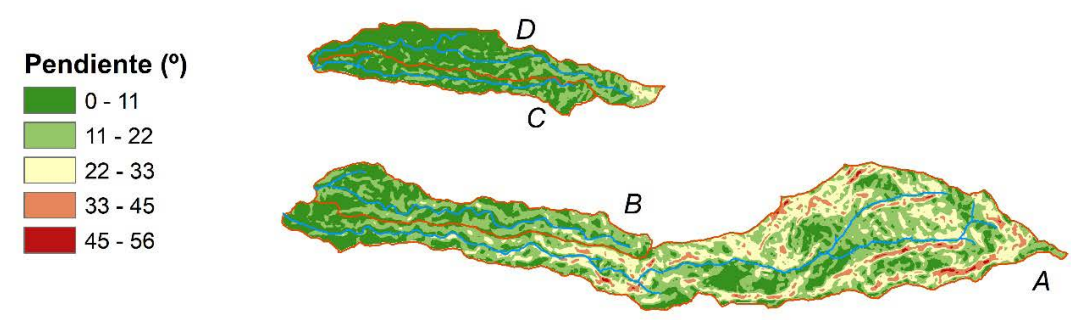

Figura 2. Descripción de densidad de vegetación (1), uso de suelo (2) y pendiente (3).

Figure 2. Description of vegetation density (1), land use (2) and slope (3).

\begin{tabular}{|c|c|c|}
\hline 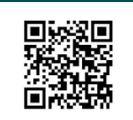 & (c) (i) $\$(0)$ & 127 \\
\hline
\end{tabular}




\section{Revista de CIENCIAS AMBIENTALES Tropical Journal of Environmental Sciences}

Revista de Ciencias Ambientales (Trop J Environ Sci)
e-ISSN: 2215-3896
(Enero-Junio, 2022).Vol 56(1): 120-137
DOI: https://doi.org/10.15359/rca.56-1.6
Open Access: www.revistas.una.ac.cr/ambientales
e-mail: revista.ambientales@una.ac.cr
Rodríguez Cueto Y., Ramón Puebla A.

Con respecto al uso de suelo, al uso forestal corresponde la mayor área (40.59\%), seguido del pastoril (27.22 \%), desnudo (20.97\%) y urbano (11.22\%); el uso menos frecuente son los invernaderos $(0.01 \%)$. Se observa que la mayor predominancia de uso forestal y suelo desnudo se encuentra en la microcuenca A y la mayor predominancia de uso pastoril se encuentra en las microcuencas C y D. Las zonas urbanas en las microcuencas A y B se ubican en la parte baja $\mathrm{y}$, en las zonas $\mathrm{C}$ y D, en la parte alta. En general, los datos de densidad de vegetación y uso de suelo clasifican la zona de estudio como rural con actividades predominantemente ganaderas y parches urbanos heterogéneamente distribuidos.

La geomorfología de la zona es muy heterogénea, el valor medio de la pendiente es $15.3^{\circ}$, con un rango entre $0.0^{\circ}-55.7^{\circ}$. La mayor parte del área total $(77.65 \%)$ abarca una pendiente entre $0.0^{\circ}-22.0^{\circ}$, mientras que pendientes entre $44.0^{\circ}-55.0^{\circ}$ comprenden un área del $0.56 \%$. Esto clasifica a la zona de estudio con una pendiente baja, con excepciones puntuales ubicadas en la microcuenca A, que coinciden con las zonas de menor vegetación, suelo desnudo y cercanas a los cuerpos de agua (Figura 2). La altitud media es de 2029 m.s.n.m. y el desnivel altitudinal es de 1454 m.s.n.m. -2 980 m.s.n.m.

\subsection{Descripción temporal y comportamiento de variables microbiológicas}

Durante el periodo en estudio (entre febrero de 2017 a marzo de 2018), la precipitación anual promedio fue de $5.1 \mathrm{~mm}$, oscilando entre $0.00-71.20 \mathrm{~mm}$ y la temperatura promedio diaria fue de $16.4^{\circ} \mathrm{C}$, con una variación entre 7.65-24.11 ${ }^{\circ} \mathrm{C}$. Tanto la precipitación como la temperatura se encuentran en el ámbito esperado para la región (IMN, 2015). El mes de mayo de 2017 presentó la máxima de precipitación y la máxima de temperatura promedio del periodo de estudio (Figura 3). La precipitación acumulada fue de $2057 \mathrm{~mm} / a n ̃ o$, que se encuentra dentro del rango esperado para zonas bioclimáticas clasificadas como bosque muy húmedo montano (2000-4000 mm/año) (Holdridge, 1967). En resumen, las condiciones climáticas durante el periodo de estudio presentan un comportamiento típico para la región, a pesar de que durante el periodo de estudio la Tormenta Tropical Nate (octubre del 2017) afectó al país.

\begin{tabular}{|c|c|c|}
\hline 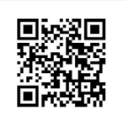 & (c) (i) (9) & 128 \\
\hline
\end{tabular}




\section{Revista de CIENCIAS AMBIENTALES Tropical Journal of Environmental Sciences}

Revista de Ciencias Ambientales (Trop J Environ Sci) e-ISSN: 2215-3896 (Enero-Junio, 2022) . Vol 56(1): 120-137 DOI: https://doi.org/10.15359/rca.56-1.6 Open Access: www.revistas.una.ac.cr/ambientales e-mail: revista.ambientales@una.ac.cr Rodríguez Cueto Y., Ramón Puebla A.

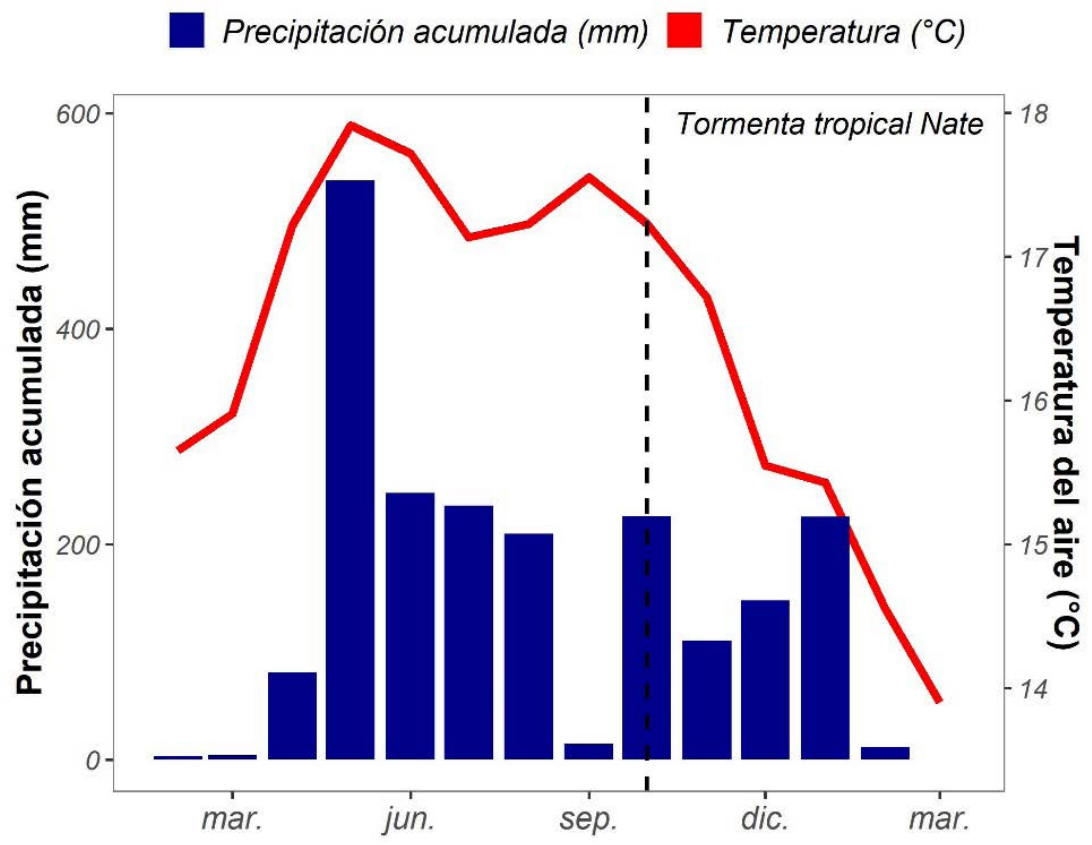

Figura 3. Climograma de la zona de estudio entre febrero del 2017 a marzo del 2018 de la precipitación mensual acumulada y la temperatura mensual promedio a partir de datos registrados por el Instituto Meteorológico Nacional. Figure 3. Climograph of the study area between February 2017 to March 2018 of the accumulated monthly precipitation and the average monthly temperature from data registered by the National Meteorological Institute.

Para Cryptosporidium sp., la concentración media fue de 0.76 (0.10-30.00) ooquistes/L. El comportamiento de este patógeno durante el muestreo no fue uniforme, solamente el $12.93 \%$ (14/108) de las muestras fueron positivas y en la zona de captación C no se detectó al parásito (Figura 4). No se encontró diferencias significativas entre la concentración de Cryptosporidium sp. $(\mathrm{p}=0.30)$ entre las zonas de captación ni entre las épocas climáticas seca y lluviosa $(\mathrm{p}=0.10)$ (Figura 4). Con respecto a Giardia sp., la concentración media fue 0.75 (0.1-10.0) quistes/L, donde un $15.93 \%(17 / 108)$ de las muestras fueron positivas. Este parásito presentó un comportamiento uniforme y se detectó en las cuatro zonas de captación. Se encontraron diferencias significativas entre las zonas de captación $(\mathrm{p}=0.09)$, pero no entre las épocas climáticas $(\mathrm{p}=0.30)$ (Figura 4).

Los colifagos somáticos presentaron una media de 108.08 (1.00-1 465.00) UFP/100 mL. El $51.86 \%(56 / 108)$ de las muestras fueron positivas, con un comportamiento uniforme en las cuatro zonas de captación (Figura 4). No hubo diferencias significativas entre las zonas de captación ( $\mathrm{p}=0.80)$; sin embargo, sí se encontraron diferencias significativas entre las épocas climáticas $(\mathrm{p}<0.001)$, contrariamente a lo observado con Cryptosporidium sp. y Giardia sp. Las concentraciones más altas de colifagos somáticos se observaron en meses de época lluviosa

\begin{tabular}{|c|c|c|}
\hline 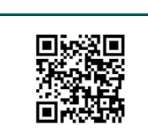 & (c) (1) () () (2) & 129 \\
\hline
\end{tabular}




\section{Revista de CIENCIAS AMBIENTALES Tropical Journal of Environmental Sciences}

Revista de Ciencias Ambientales (Trop J Environ Sci) e-ISSN: 2215-3896 (Enero-Junio, 2022) . Vol 56(1): 120-137 DOI: https://doi.org/10.15359/rca.56-1.6 Open Access: www.revistas.una.ac.cr/ambientales e-mail: revista.ambientales@una.ac.cr Rodríguez Cueto Y., Ramón Puebla A.

(junio, julio y agosto). Adicionalmente, en mayo, mes de máxima precipitación, no se observó una concentración particularmente elevada de este indicador (Figura 4).

E. coli presentó una media de 8832 (170-240 000) NMP/100 mL, el $100 \%(108 / 108)$ de las muestras fueron positivas, con un comportamiento similar en todas las zonas de captación. Se encontraron diferencias significativas entre las áreas de estudio $(\mathrm{p}=0.05)$ y las épocas climáticas $(\mathrm{p}<0.001)$ (Figura 4).

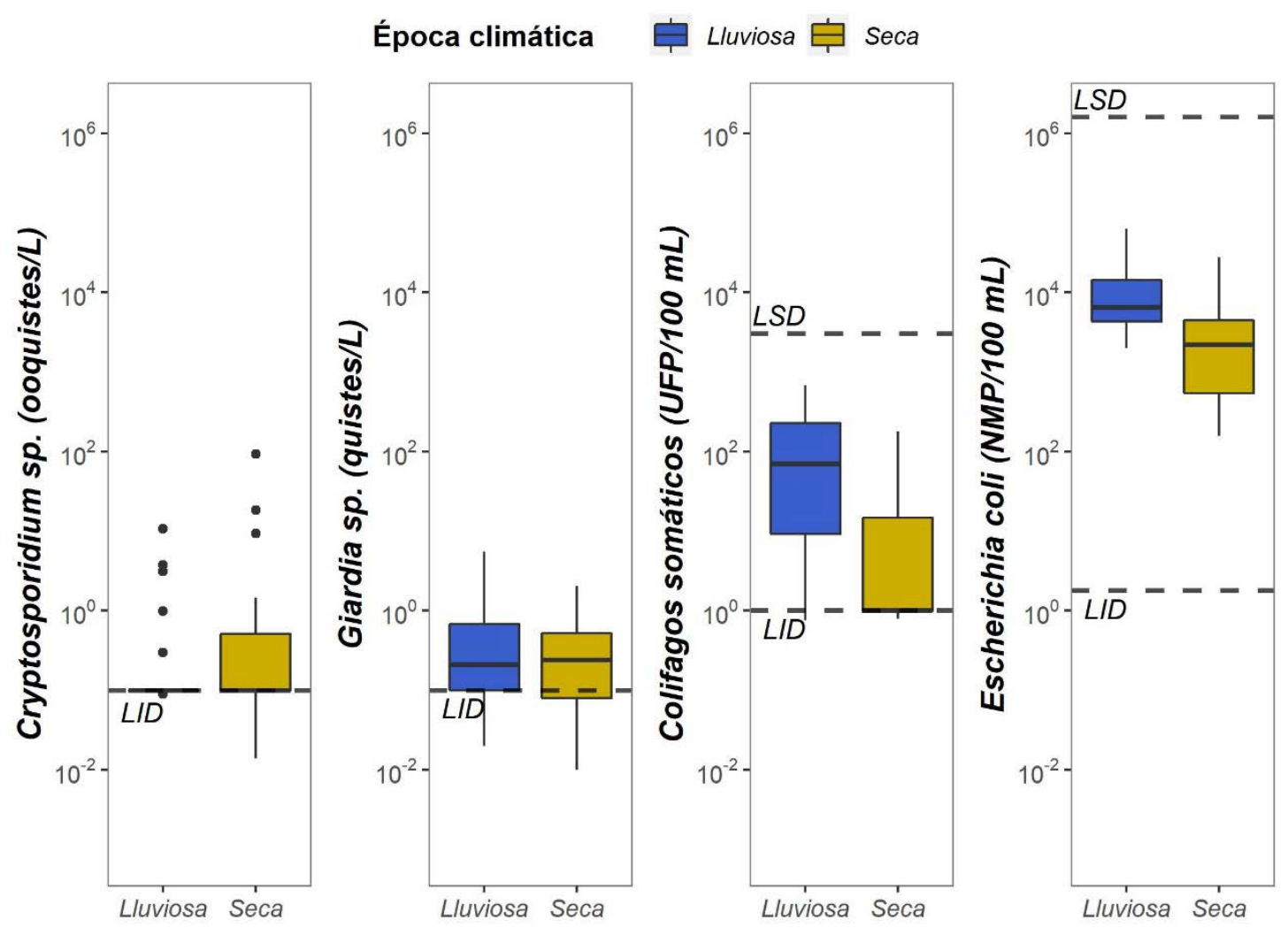

Figura 4. Concentraciones de Cryptosporidium sp., Giardia sp., colifagos somáticos y E. coli por época climática. LID: límite inferior de detección, LSD: límite superior de detección.

Figure 4. Seasonal concentrations of Cryptosporidium sp., Giardia sp., somatic coliphages, and E. coli. LID: lower limit of detection, LSD: upper limit of detection.

\subsection{Relación de variables microbiológicas y precipitación diaria acumulada $24 \mathrm{~h}$ antes de tomar la muestra \\ La precipitación diaria acumulada $24 \mathrm{~h}$ antes del muestreo fue dividida en cuatro categorías: débil $(\geq 2 \mathrm{~mm} / \mathrm{h}$ ), moderada $(>2-\leq 15 \mathrm{~mm} / \mathrm{h})$, fuerte $(>15-\leq 30 \mathrm{~mm} / \mathrm{h})$ y muy fuerte $(>30-\leq 60$ $\mathrm{mm} / \mathrm{h}$ ) y se analizó junto con la concentración de microorganismos utilizando un modelo de regresión de Poisson, lo cual mostró asociaciones estadísticas significativas (Cuadro 1). En el}

\begin{tabular}{|c|c|c|}
\hline 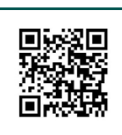 & (c) (1) (5) (2) & 130 \\
\hline
\end{tabular}




\section{Revista de CIENCIAS AMBIENTALES Tropical Journal of Environmental Sciences}

Revista de Ciencias Ambientales (Trop J Environ Sci) e-ISSN: 2215-3896 (Enero-Junio, 2022) . Vol 56(1): 120-137 DOI: https://doi.org/10.15359/rca.56-1.6 Open Access: www.revistas.una.ac.cr/ambientales e-mail: revista.ambientales@una.ac.cr Rodríguez Cueto Y., Ramón Puebla A.

caso de Cryptosporidium sp., hay mayor razón de probabilidad de encontrar ooquistes cuando la precipitación es débil. Para Giardia sp., se observa que la razón de probabilidad de encontrar quistes es mayor cuando la intensidad de la lluvia se clasifica como débil y como muy fuerte. Los colifagos somáticos a diferencia de los microorganismos anteriores presentan un aumento de la razón de probabilidad de ser detectados conforme aumenta la intensidad de la precipitación, excepto cuando la precipitación es muy fuerte, donde la razón de probabilidad es menor en comparación con el resto de los escenarios. Por último, para E. coli, se observa un aumento de la razón de probabilidad de detección, conforme aumenta la intensidad de la precipitación, con excepción de los escenarios de precipitación débil y moderada, donde el primero presenta una mayor razón de probabilidad.

Cuadro 1. Análisis de regresión de Poisson de la relación entre de la concentración de Cryptosporidium sp., Giardia sp., colifagos somáticos y E. coli; $y$ la intensidad de precipitación $24 \mathrm{~h}$ antes del muestreo.

Table 1. Poisson regression analysis of the intensity of precipitation $24 \mathrm{~h}$ before sampling and its relationship with the concentrations of Cryptosporidium sp., Giardia sp., Somatic coliphages and E. coli.

\begin{tabular}{|c|c|c|c|c|}
\hline Intensidad precipitación & OR & Límite inferior $2.5 \%$ & Límite superior $97.5 \%$ & $\mathbf{p}$ \\
\hline \multicolumn{5}{|c|}{ Cryptosporidium sp. - Bondad del ajuste $=0.18$, prueba de dispersión $=0.06$} \\
\hline Débil & 10.95 & 4.92 & 30.98 & $<0.001$ \\
\hline Moderada & 0.74 & 0.18 & 2.82 & 0.66 \\
\hline Fuerte & 0.55 & 0.02 & 3.81 & 0.62 \\
\hline Muy Fuerte & 0.55 & 0.02 & 3.81 & 0.62 \\
\hline \multicolumn{5}{|c|}{ Giardia sp. - Bondad del ajuste $=0.27$, prueba de dispersión $=0.07$} \\
\hline Débil & 3.84 & 1.73 & 10.1 & 0.002 \\
\hline Moderada & 0.75 & 0.21 & 2.52 & 0.65 \\
\hline Fuerte & 0.73 & 0.06 & 3.77 & 0.75 \\
\hline Muy Fuerte & 6.08 & 2.31 & 17.51 & $<0.001$ \\
\hline \multicolumn{5}{|c|}{ Colifagos somáticos - Bondad del ajuste $=0.34$, prueba de dispersión $=0.14$} \\
\hline Débil & 6.9 & 6.31 & 7.56 & $<0.001$ \\
\hline Moderada & 8.2 & 7.5 & 8.98 & $<0.001$ \\
\hline Fuerte & 11.43 & 10.4 & 12.59 & $<0.001$ \\
\hline Muy Fuerte & 2.81 & 2.49 & 3.18 & $<0.001$ \\
\hline \multicolumn{5}{|c|}{ Escherichia coli - Bondad del ajuste $=0.51$, prueba de dispersión $=0.15$} \\
\hline Débil & 1.8 & 1.79 & 1.81 & $<0.001$ \\
\hline Moderada & 1.08 & 1.07 & 1.09 & $<0.001$ \\
\hline Fuerte & 2.29 & 2.27 & 2.31 & $<0.001$ \\
\hline Muy Fuerte & 9.06 & 9.00 & 9.12 & $<0.001$ \\
\hline
\end{tabular}

\section{Discusión}

Este trabajo demuestra que el recurso hídrico de la zona de estudio se encuentra con alta vulnerabilidad. El área geográfica donde se ubican las microcuencas se clasifica como rural y presenta parches urbanos distribuidos heterogéneamente, lo cual implica una amenaza tanto de riesgos urbanos como rurales, manifestados por las altas concentraciones de los patógenos e

\begin{tabular}{|c|c|c|c|c|c|}
\hline 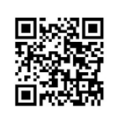 & (c) (i) () () & $\overbrace{\text { AMBEENTALS }}$ & $\begin{array}{l}\frac{9 \%}{2} \\
\frac{2}{2} \\
\text { euna }\end{array}$ & $\frac{\text { UNA }}{\frac{\text { UNIIERISAD }}{\text { UASEIONAL }}}$ & 131 \\
\hline
\end{tabular}




\section{Revista de CIENCIAS AMBIENTALES Tropical Journal of Environmental Sciences}

Revista de Ciencias Ambientales (Trop J Environ Sci) e-ISSN: 2215-3896 (Enero-Junio, 2022) . Vol 56(1): 120-137 DOI: https://doi.org/10.15359/rca.56-1.6 Open Access: www.revistas.una.ac.cr/ambientales e-mail: revista.ambientales@una.ac.cr Rodríguez Cueto Y., Ramón Puebla A.

indicadores de contaminación fecal encontrados (Figuras 2 y 4). En estudios previos se describió que el uso de suelo pastoril y agrícola tienen la capacidad de retener contaminantes microbiológicos en mayor proporción que el uso urbano (Bergion et al., 2017), lo cual se pudo corroborar con los niveles más bajos de microorganismos patógenos en las microcuencas $\mathrm{C}$ y $\mathrm{D}$, en contraste con A y B, que son las que presentan mayor cantidad de parches urbanos (Figura 2).

A pesar de que no se determinaron descargas puntuales de aguas residuales, se observa el riesgo latente de estas, principalmente en las microcuencas $\mathrm{A}$ y $\mathrm{B}$, que tienen parches de uso de suelo urbano cerca de la zona de captación de agua para consumo humano (Figura 2); la influencia negativa del uso de suelo urbano sobre la calidad del agua ha sido ampliamente demostrada, principalmente las descargas puntuales de aguas residuales en los cuerpos de agua superficiales (Schreiber et al., 2015). Con respecto a la densidad de vegetación, aunque las microcuencas A y B presentan una alta proporción de bosque secundario y terciario, son las más vulnerables a contaminación, pues mostraron un bajo NDVI, pendientes más pronunciadas y concentraciones más altas de variables microbiológicas, a diferencia de las microcuencas $\mathrm{C} \mathrm{y}$ D (NDVI más alto, pendientes menores y niveles de contaminación microbiológica menores) (Figuras 2 y 4). Adicionalmente, en el caso particular de Cryptosporidium sp. los niveles altos de NDVI han sido relacionados con altas concentraciones de este protozoario, recomendándose su aplicación para evaluar el potencial riesgo de infección (Jagai et al., 2009).

Con respecto al resto de variables microbiológicas se presentaron diferencias estadísticamente significativas entre las microcuencas para Giardia sp. y E. coli, lo cual puede indicar que las diferencias entre las condiciones espaciales de las distintas microcuencas tienen influencia sobre las concentraciones de microorganismos en la zona de captación (punto más bajo de cada microcuenca).

En referencia a la influencia climática en el comportamiento de las variables microbiológicas analizadas, se encontraron diferencias significativas entre la época lluviosa y la época seca para colifagos somáticos y E. coli. Estas observaciones coinciden con lo previamente informado, donde el aumento en la precipitación tiene una estrecha relación con el aumento en la concentración de estos microorganismos; además, se ha descrito que el incremento en la precipitación tiende a tener una mayor capacidad de arrastrar partículas sedimentables y microorganismos hacia los cuerpos de agua (Cizek et al., 2008). Este patrón se observó en este estudio, donde los microorganismos indicadores se encontraron en mayor concentración en época lluviosa (Figura 4). Contrariamente, la detección de Cryptosporidium sp. y Giardia sp. fue más frecuente en época seca; sin embargo, no hay diferencias estadísticamente significativas entre ambas épocas climáticas, por lo cual, es posible que la principal fuente de estos protozoarios sean descargas puntuales, mientras que su menor concentración durante la época lluviosa se podría atribuir a su dilución tras el aumento del caudal producido por la precipitación, lo cual concuerda con lo descrito por Schreiber et al., (2015) que altas concentraciones de parásitos como Cryptosporidium sp. y Giardia sp. pueden asociarse principalmente a descargas directas de aguas residuales. En resumen, es probable que la fuente contaminación entre Cryptosporidium sp. y Giardia sp.

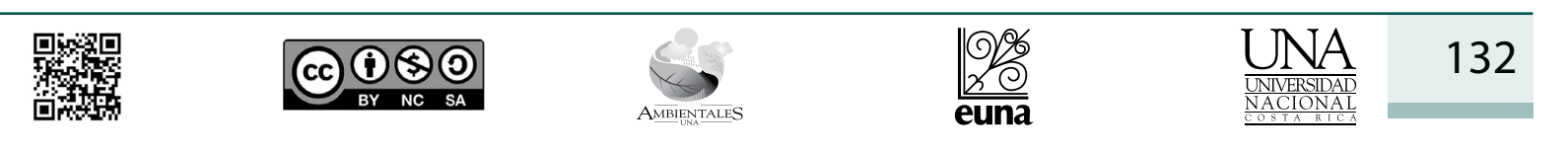




\section{Revista de CIENCIAS AMBIENTALES Tropical Journal of Environmental Sciences}

Revista de Ciencias Ambientales (Trop J Environ Sci) e-ISSN: 2215-3896 (Enero-Junio, 2022) . Vol 56(1): 120-137 DOI: https://doi.org/10.15359/rca.56-1.6 Open Access: www.revistas.una.ac.cr/ambientales e-mail: revista.ambientales@una.ac.cr Rodríguez Cueto Y., Ramón Puebla A.

sea diferente a la de colifagos somáticos y E. coli, puesto que no presentan el mismo patrón estacional; por ello, la influencia de patrones temporales, como el clima, o espaciales como el uso de suelo, la vegetación y geomorfología tienen una influencia diferente entre los microorganismos estudiados (Figuras 2, 3 y 4).

Aunado a lo anterior, en la mayoría de los casos se observa que la intensidad de precipitación 24 horas antes del muestreo presenta una asociación importante con la concentración de los microorganismos encontrados (Cuadro 1). Para Cryptosporidium sp. y Giardia sp., se observa que hay mayor probabilidad de detectarlos si la precipitación tiene una intensidad débil, a diferencia de E. coli y colifagos somáticos, donde el aumento en las concentraciones se asocia con mayor intensidad de la precipitación. Lo anterior concuerda con los resultados de Schreiber et al., (2015), donde la presencia de parásitos se asocia con fuentes de contaminación puntuales, como ya se mencionó, levemente influenciables por las condiciones climáticas, mientras que los indicadores fecales se asocian a fuentes de contaminación difusas, altamente influenciables por condiciones climáticas. Al respecto, se ha descrito que hay una fuerte asociación entre la concentración bacterias y colifagos somáticos; conforme aumenta la intensidad de la precipitación antes de la toma de la muestra, más elevada es la concentración detectada de ambos microorganismos, lo cual concuerda con los resultados encontrados en el presente estudio (Cuadro 1) (Bergion et al., 2017; Cizek et al., 2008).

A pesar de la influencia de la precipitación en E. coli y colifagos somáticos, estos no presentan una asociación entre la detección de uno con respecto a otro. Estudios previos han descrito que los colifagos somáticos presentan una mayor asociación a virus entéricos que a indicadores fecales como E. coli, por lo cual, podrían ser buenos indicadores de contaminación fecal viral (Chacón et al., 2020; McMinn et al., 2017).

\section{Conclusiones}

Este trabajo explora la importancia del análisis individual de cada microorganismo, estacional por épocas del año, puntual previo al muestreo y espacial. Como parte de ello se pueden destacar varios puntos.

1. La presencia de E. coli no se asocia con la presencia de protozoarios y virus, lo cual implica que no puede ser un indicador de calidad microbiológica del agua para consumo por sí solo

2. La relevancia de considerar el comportamiento estacional y el diario para determinar la relación entre las concentraciones de los microorganismos y la precipitación; debido a que el estacional muestra el comportamiento general en relación con las épocas climáticas, en este caso, para colifagos somáticos y E. coli las concentraciones más altas se encuentran en meses de alta precipitación, mientras se observa un comportamiento inverso para Cryptosporidium sp. y Giardia sp., una limitación del presente estudio en este aspecto fue no contar con datos históricos de la zona para determinar si el comportamiento climático fue típico

\begin{tabular}{|c|c|c|c|c|c|}
\hline 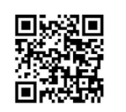 & (c) (1) () (2) & $\overbrace{\text { AMBENTILES }}$ & $\frac{1 \%}{2 \%}$ & 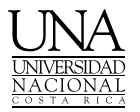 & 133 \\
\hline
\end{tabular}




\section{Revista de CIENCIAS AMBIENTALES Tropical Journal of Environmental Sciences}

Revista de Ciencias Ambientales (Trop J Environ Sci)
e-ISSN: 2215-3896
(Enero-Junio, 2022). Vol 56(1): 120-137
DOI: https://doi.org/10.15359/rca.56-1.6
Open Access: www.revistas.una.ac.cr/ambientales
e-mail: revista.ambientales@una.ac.cr
Rodríguez Cueto Y., Ramón Puebla A.

en las microcuencas. El comportamiento diario muestra la influencia puntual de la precipitación en el momento de la toma de la muestra para los cuatro microorganismos, lo cual puede resultar de utilidad para la gestión del recurso hídrico, por ejemplo, en el caso de los protozoarios su detección es menor cuando 24 horas antes del muestreo se ha presentado una precipitación de alta o muy alta intensidad, lo cual se puede deber al comportamiento natural de los microorganismos o la modificación ambiental producto de la precipitación como dilución, aumento de escorrentía, entre otros.

3. Las condiciones ambientales como la cobertura de vegetación, el uso de suelo, el tipo de suelo y la pendiente determinan el transporte y frecuencia de contaminantes microbiológicos en los cuerpos de agua. Los aspectos anteriores son relevantes considerando la administración del recurso hídrico en un escenario de cambio climático, donde los regímenes de precipitación, aunado a procesos de contaminación ambiental pueden magnificar la presencia de patógenos en el agua para consumo humano, de ahí la importancia de la inclusión del análisis temporal (climático) y espacial en el monitoreo de las fuentes de agua.

\section{Conflicto de intereses}

Las personas autoras declaran que han cumplido totalmente con todos los requisitos éticos y legales pertinentes, tanto durante el estudio como en la producción del manuscrito; que no hay conflictos de intereses de ningún tipo; que todas las fuentes financieras se mencionan completa y claramente en la sección de agradecimientos; y que están totalmente de acuerdo con la versión final editada del artículo.

\section{Agradecimientos}

Se agradece a la Vicerrectoría de Investigación de la Universidad de Costa Rica por su apoyo para la realización de este trabajo y al AyA por su colaboración durante la recolección de las muestras. También se agradece al editor y a las personas revisoras anónimas por los comentarios realizados a la versión final del escrito.

\section{Referencias}

Baird, R., Rice, C., \& Eaton, A. (2017). Standard Methods for the examination of water and wastewater (23rd ed). American Public Health Association. http://dspace.uniten.edu.my/ handle/123456789/14241

Bergion, V., Sokolova, E., Åström, J., Lindhe, A., Sörén, K., \& Rosén, L. (2017). Hydrological modelling in a drinking water catchment area as a means of evaluating pathogen risk reduction. Journal of Hydrology, 544, 74-85. https://doi.org/10.1016/j.jhydrol.2016.11.011

\begin{tabular}{|c|c|c|c|c|}
\hline 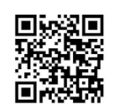 & (c) (1) () (2) & $\underset{\text { AMBENENALIES }}{8}$ & $\frac{1 \%}{2 \%}$ & 134 \\
\hline
\end{tabular}




\section{Revista de CIENCIAS AMBIENTALES Tropical Journal of Environmental Sciences}

Revista de Ciencias Ambientales (Trop J Environ Sci)
e-ISSN: 2215-3896
(Enero-Junio, 2022).Vol 56(1): 120-137
DOI: https://doi.org/10.15359/rca.56-1.6
Open Access: www.revistas.una.ac.cr/ambientales
e-mail: revista.ambientales@una.ac.cr
Rodríguez Cueto Y., Ramón Puebla A.

Bertuzzo, E., \& Mari, L. (2017). Hydrology, water resources and the epidemiology of water-related diseases. Advances in Water Resources, 108, 329-331. https://doi.org/10.1016/j. advwatres.2017.09.011

Cabello-Piñar, J., Alcaraz-Segura, D., Reyes-Díez, A., Lourenço, P., Requena, J. M., Bonache, J., Castillo, P., Valencia, S., Naya, J., Ramírez, L., \& Serrada, J. (2016). Sistema para el Seguimiento del funcionamiento de ecosistemas en la Red de Parques Nacionales de España mediante Teledetección. Revista de Teledetección, 46, 119-131. https://doi.org/10.4995/raet.2016.5731

Chacón, L., Barrantes, K., Santamaría-Ulloa, C., Solano, M., Reyes, L., Taylor, L., Valiente, C., Symonds, E. M., \& Achí, R. (2020). A somatic coliphage threshold approach to improve the management of activated sludge wastewater treatment plant effluents in resource-limited regions. Applied and Environmental Microbiology, 86(17), 1-16. https://doi.org/10.1128/AEM.00616-20

Chalmers, R. M. (2014). Cryptosporidium. In Microbiology of Waterborne Diseases: Microbiological Aspects and Risks (2da ed.). https://doi.org/10.1016/B978-0-12-415846-7.00016-0

Cizek, A. R., Characklis, G. W., Krometis, L.A., Hayes, J. A., Simmons, O. D., Di Lonardo, S., Alderisio, K. A., \& Sobsey, M. D. (2008). Comparing the partitioning behavior of Giardia and Cryptosporidium with that of indicator organisms in stormwater runoff. Water Research, 42(17), 4421-4438. https://doi.org/10.1016/j.watres.2008.06.020

Dheenan, P. S., Jha, D. K., Das, A. K., Vinithkumar, N. V., Devi, M. P., \& Kirubagaran, R. (2016). Geographic information systems and multivariate analysis to evaluate fecal bacterial pollution in coastal waters of Andaman, India. Environmental Pollution, 214, 45-53. https://doi.org/10.1016/j.envpol.2016.03.065

Helsel, D. R. (2011). Statistics for Censored Environmental Data using Minitab and R. John Wiley \& Sons. https://doi.org/10.1002/9781118162729

Holdridge, L. R. (1967). Life zone ecology. Tropical Science Center. http://reddcr.go.cr/sites/default/files/centro-de-documentacion/holdridge_1966_-_life_zone_ecology.pdf

Instituto Meteorológico Nacional. (2015). Pronostico Climático 2015. https://www.imn.ac. $\mathrm{cr} /$ documents/10179/29806/Perspectiva+Clim\%C3\%A1tica+Anual+2015+para+CR/ f5fe3229-4a48-4e11-8360-933b4faf6a81?version=1.0

Jagai, J. S., Castronovo, D. A., Monchak, J., \& Naumova, E. N. (2009). Seasonality of cryptosporidiosis: A meta-analysis approach. Environmental Research, 109(4), 465-478. https://doi. org/10.1016/j.envres.2009.02.008

\begin{tabular}{|c|c|c|c|c|c|}
\hline 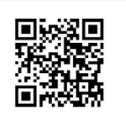 & (c) & $\overbrace{\text { AMEIENTILES }}$ & $\frac{9 \%}{20}$ & $\frac{\text { UNA }}{\frac{\text { UNIVERIDAD }}{\text { NACIONAL }}}$ & 135 \\
\hline
\end{tabular}




\section{Revista de CIENCIAS AMBIENTALES Tropical Journal of Environmental Sciences}

$$
\begin{array}{r}
\text { Revista de Ciencias Ambientales (Trop J Environ Sci) } \\
\text { e-ISSN: 2215-3896 } \\
\text { (Enero-Junio, 2022). Vol 56(1): 120-137 } \\
\text { DOI: https://doi.org/10.15359/rca.56-1.6 } \\
\text { Open Access: www.revistas.una.ac.cr/ambientales } \\
\text { e-mail: revista.ambientales@una.ac.cr } \\
\text { Rodríguez Cueto Y., Ramón Puebla A. }
\end{array}
$$

Karanis, P., Sotiriadou, I., Kartashev, V., Kourenti, C., Tsvetkova, N., \& Stojanova, K. (2006). Occurrence of Giardia and Cryptosporidium in water supplies of Russia and Bulgaria. Environmental Research, 102(3), 260-271. https://doi.org/10.1016/j.envres.2006.05.005

Lal, A., Baker, M. G., Hales, S., \& French, N. P. (2013). Potential effects of global environmental changes on cryptosporidiosis and giardiasis transmission. Trends in Parasitology, 29(2), 83-90. https://doi.org/10.1016/j.pt.2012.10.005

Lee, L. (2020). NADA: Nondetects and Data Analysis for Environmental Data. [Software]. https://cran.r-project.org/web/packages/NADA/index.html

McMinn, B. R., Ashbolt, N. J., \& Korajkic, A. (2017). Bacteriophages as indicators of faecal pollution and enteric virus removal. Letters in Applied Microbiology, 65(1), 11-26. https://doi.org/10.1111/lam.12736

Morales, E., Solano, M., Morales, R., Reyes, L., Barrantes, K., Achí, R., \& Chacón, L. (2019). Evaluation of climatic seasonal influence on the quality of water for human consumption in a San José (Costa Rica) supply system, 2017-2018. Revista costarricense de Salud Pública, 28(1), 7787. https://www.scielo.sa.cr/scielo.php?pid=S1409-14292019000100048\&script=sci_abstract

Ortiz-Malavasi, E. (2014). Atlas de Costa Rica. Instituto Tecnológico de Costa Rica. https://revistas.tec.ac.cr/index.php/investiga_tec/article/view/2330

Pettorelli, N., Vik, J. O., Mysterud, A., Gaillard, J. M., Tucker, C. J., \& Stenseth, N. C. (2005). Using the satellite-derived NDVI to assess ecological responses to environmental change. Trends in Ecology and Evolution, 20(9), 503-510. https://doi.org/10.1016/j.tree.2005.05.011

Robertson, L. J. (2014). Giardia duodenalis. In Microbiology of Waterborne Diseases: Microbiological Aspects and Risks. Academic Press. 375-405. https://doi.org/10.1016/ B978-0-12-415846-7.00019-6

Schreiber, C., Rechenburg, A., Rind, E., \& Kistemann, T. (2015). The impact of land use on microbial surface water pollution. International Journal of Hygiene and Environmental Health, 218(2), 181-187. https://doi.org/10.1016/j.ijheh.2014.09.006

Solano Barquero, M., Chacón Jiménez, L. M., Barrantes Jiménez, K., \& Achí Araya, R. (2012).

\begin{tabular}{|c|c|c|c|c|}
\hline 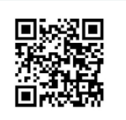 & (c) (i) $\$(9)$ & 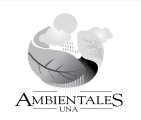 & $\frac{1 \% \%}{\text { euna }}$ & 136 \\
\hline
\end{tabular}
Implementación de dos métodos de recuento en placa para la detección de colifagos somáticos, aportes a las metodologías estándar. Revista Peruana de Biología, 19(3), 335-340. https://doi.org/10.15381/rpb.v19i3.1050

Strachan, N. J. C., Dunn, G. M., Locking, M. E., Reid, T. M. S., \& Ogden, I. D. (2006). Escherichia coli O157: Burger bug or environmental pathogen?. International Journal of Food Microbiology, 112(2), 129-137. https://doi.org/10.1016/j.ijfoodmicro.2006.06.021 


\section{Revista de CIENCIAS AMBIENTALES

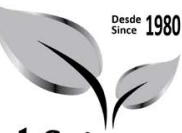 Tropical Journal of Environmental Sciences}

$$
\begin{array}{r}
\text { Revista de Ciencias Ambientales (Trop J Environ Sci) } \\
\text { e-ISSN: 2215-3896 } \\
\text { (Enero-Junio, 2022). Vol 56(1): 120-137 } \\
\text { DOI: https://doi.org/10.15359/rca.56-1.6 } \\
\text { Open Access: www.revistas.una.ac.cr/ambientales } \\
\text { e-mail: revista.ambientales@una.ac.cr } \\
\text { Rodríguez Cueto Y., Ramón Puebla A. }
\end{array}
$$

US Environmental Protection Agency. (2005). Method 1623: Cryptosporidium and Giardia in Water by Filtration/IMS/FA. Office of Watter, US

United States Geological Survey. (2021). NDVI, the Foundation for Remote Sensing Phenology. https://www.usgs.gov/core-science-systems/eros/phenology/science/ndvi-foundation-remote-sensing-phenology?qt-science_center_objects=0\#qt-science_center_objects

Valle Júnior, R. F. do, Siqueira, H. E., Valera, C. A., Oliveira, C. F., Sanches Fernandes, L. F., Moura, J. P., \& Pacheco, F. A. L. (2019). Diagnosis of degraded pastures using an improved NDVI-based remote sensing approach: An application to the Environmental Protection Area of Uberaba River Basin (Minas Gerais, Brazil). Remote Sensing Applications: Society and Environment. 14, 20-33. https://doi.org/10.1016/j.rsase.2019.02.001

World Health Organization. (2017). Guidelines for drinking-water quality (4th ed.). World Heal-

\begin{tabular}{|c|c|c|c|c|c|}
\hline 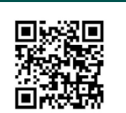 & (c) () () & $\overbrace{\text { AMBENTALS }}^{6}$ & $\begin{array}{l}\frac{O \%}{6} \\
\frac{2}{2} \\
\text { euna }\end{array}$ & $\frac{\text { UNA }}{\frac{\text { UNIVERSIDAD }}{\text { UACIONAL }}}$ & 137 \\
\hline
\end{tabular}
th Organization. https://www.joinforwater.ngo/sites/default/files/library_assets/351_WHO_ E13_guidelines_drinking-water.pdf

Intergovernmental Panel on Climate Change. (2014). Summary for Policymakers. In Climate Change 2013 - The Physical Science Basis: Working Group I Contribution to the Fifth Assessment Report of the Intergovernmental Panel on Climate Change. 1-30. Cambridge University Press. https://doi.org/10.1017/CBO9781107415324.004 